\title{
Molecular Structural Characteristics of Quinoa Starch
}

\author{
Katsumi Watanabe*, Ng Lai Peng, Hanjun TAng and Toshio Mitsunaga \\ Department of Food and Nutrition, Faculty of Agriculture, Kinki University, Naka-machi 3327-204, Nara 631-8505, Japan
}

Received June 9, 2006; Accepted October 30, 2006

\begin{abstract}
The structural properties of starches isolated from five quinoa lines were characterized. All quinoa starches used in the study exhibited the A-type of diff raction pattern. The starches were waxy, with low amylose contents $(5.2$ to $10.9 \%$ ) and high degrees of relative crystallinity (39.0 to $43.0 \%)$. Quinoa amylose molecules had a large number of branched chains, and the $\lambda$ max (maximum absorption wavelength) and $\mathrm{BV}$ (blue value at $680 \mathrm{~nm}$ ) of quinoa am ylose molecules were low compared to barley amylose molecules. On the other hand, quinoa amylopectin molecules had a large proportion of LC (longest chain) with a high $\lambda$ max and BV. These structural characteristics of quinoa starches suggested that quinoa could be used as a novel food source.
\end{abstract}

Keywords: molecular structure, quinoa, starch

\section{Introduction}

Quinoa (Chenopodium quinoa Willd) is a pseudocereal native to South America. Recently, it has attracted renewed attention because of the high nutritional value of its protein and its suppressive effect on increased blood pressure (Ranhotra et al., 1993; Ogawa et al., 2001). Quinoa protein has a high level of lysine, and its amino acid score is higher than that of cereals such as rice and wheat (Watanabe et al., 2003). Furthermore, quinoa protein contains significant amounts of albumin and globulin, and, unlike most cereals, has little prolamin and glutelin (Watanabe et al., 2003). This suggests that quinoa could be used as an alternative for individuals allergic to cereals. Starch is the major component of quinoa, comprising $60 \%$ of the seeds (Ando et al., 2002). Thus, it is important to elucidate starch characteristics in order to utilize quinoa as a food source. There are various reports on quinoa starch (Atwell et al., 1983; A hamed et al., 1996). Recently, Lindeboom et al. (2005) have reported on differences in functional and physicochemical characteristics, including gelatinization, retrogradation, pasting and swelling properties, among the starches isolated from eight quinoa lines. However, a comparison of the structural properties such as the degree of polymerization, chain-length distribution and crystallinity among starches from different varieties has not yet been reported. Thus, the molecular structural properties of starches from five quinoa lines were investigated and compared with those of other plant species to discuss the effective utilization of quinoa as a food source in this study.

\footnotetext{
* To whom correspondence should be addressed.
}

E-mail: kwatanabe@nara.kindai.ac.jp

\section{Materials and Methods}

Materials Five quinoa lines (Real, Sajama, Kanccola, Blanca, Rosada) were obtained from Asai Co. Ltd. (Nagoya, Japan).

Starch isolation Quinoa seeds were powdered with an electric mill. Starch was isolated from the powdered seeds by the alkali method (Takeda et al., 1999). Amylose and amylopectin were prepared from the isolated starches according to the method of Takeda et al. (1986).

$X$ ray diffraction of starch granules X-ray diffraction was performed using a Rint-2500 X-ray diffractometer (Rigaku Denki Co. Ltd., Tokyo, Japan) operating at $40 \mathrm{kV}$ and $80 \mathrm{~mA}$. Diffractograms were obtained from $4^{\circ} 2 \theta$ to $40^{\circ} 2 \theta$ with a scanning speed of $8^{\circ} / \mathrm{min}$ and a scanning step of $0.02^{\circ} 2 \theta$. The degree of relative crystallinity for the starch granules was calculated as the ratio of the areas of crystalline to amorphous regions on the X-ray diffractograms using Hermans' method (Nara et al., 1978).

Analysis of fine structures of starches Iodine absorption spectra were measured by the method described previously (Takeda et al., 1983). The number-average degrees of polymerization (DPn) in the amylose and amylopectin molecules were determined by the modified ParkJohnson method (Hizuruki et al., 1981). The average chain length (CL) was determined by the same method after isoamylolysis of the molecules (Tang et al., 2001a, b). The average number of chains per molecule (NC) was the value of ((DPn/CL)-1) (Tang et al., 2001a).

Chain-length distribution in the amylose and amylopectin molecules was analyzed on two sequentially linked columns (TSK gel G3000SW + TSK gel G2000SW, 7.5 mm $\times 60 \mathrm{~cm})$ at $35^{\circ} \mathrm{C}$ using the Shimazu LC-6A system equipped with Shimazu RID-2A and Shimazu C-R6A (Shimazu Co. Ltd., Kyoto, Japan) as reported previously (Tang et al., 2001a, b). The total carbohydrate amount was deter- 
mined by the phenol-sulfuric acid method (Dobois et al., 1956).

\section{Results}

$X$-ray diffraction of starch granules Figure 1 shows the X-ray diffraction patterns of starch granules from the five quinoa lines. The starch granules exhibited the Atype of diffraction pattern common to most cereal starches (Atwell et al., 1983; Zobel 1988). The degrees of relative crystallinity of the starch granules were 39.0, 41.2, 42.8, 40.0, and 43.0\% for Real, Sajama, Kanccola, Blanca, and Rosada, respectively.

Properties of amylose and amylopectin molecules The properties of the amylose and amylopectin molecules from the five quinoa lines are presented in Tables 1 and 2 . The $\lambda$ max (maximum absorption wavelength) and BV (blue value at $680 \mathrm{~nm}$ ) of the amylose molecules were 648 to $650 \mathrm{~nm}$ and 0.998 to 1.101 , respectively. The DPn and CL of the amylose molecules were 822 to 1054 and 98 to 119, suggesting that quinoa amylose had a number of branched chains ( $\mathrm{NC}=6$ to 9 ). On the other hand, the $\lambda$ max and BV of the amylopectin molecules were 589 to 590 $\mathrm{nm}$ and 0.190 to 0.210 , respectively. The DPn and CL were 3403 to 4752 and 18 to 20.

The chain-length distribution of the amyloses is shown in Fig. 2. The amylose molecules contained C-chains (CC) which characterize the molecular structure of amylose (Takeda et al., 1986). However, short chains were also found in the amylose molecules from quinoa starches. This result suggested that the quinoa amylose molecule had branched chains. Figure 3 shows the chainlength distribution of the amylopectins. The longest chains (LC) were found in the amylopectin molecules.
The carbohydrate proportion (weight\%) of LC was 15.4 to 19.9\%. The value was higher than that of barley amylopectin molecules (Tang et al., 2001a, b). The major peaks were divided into four fractions, F.1 to F.4. F.1 was

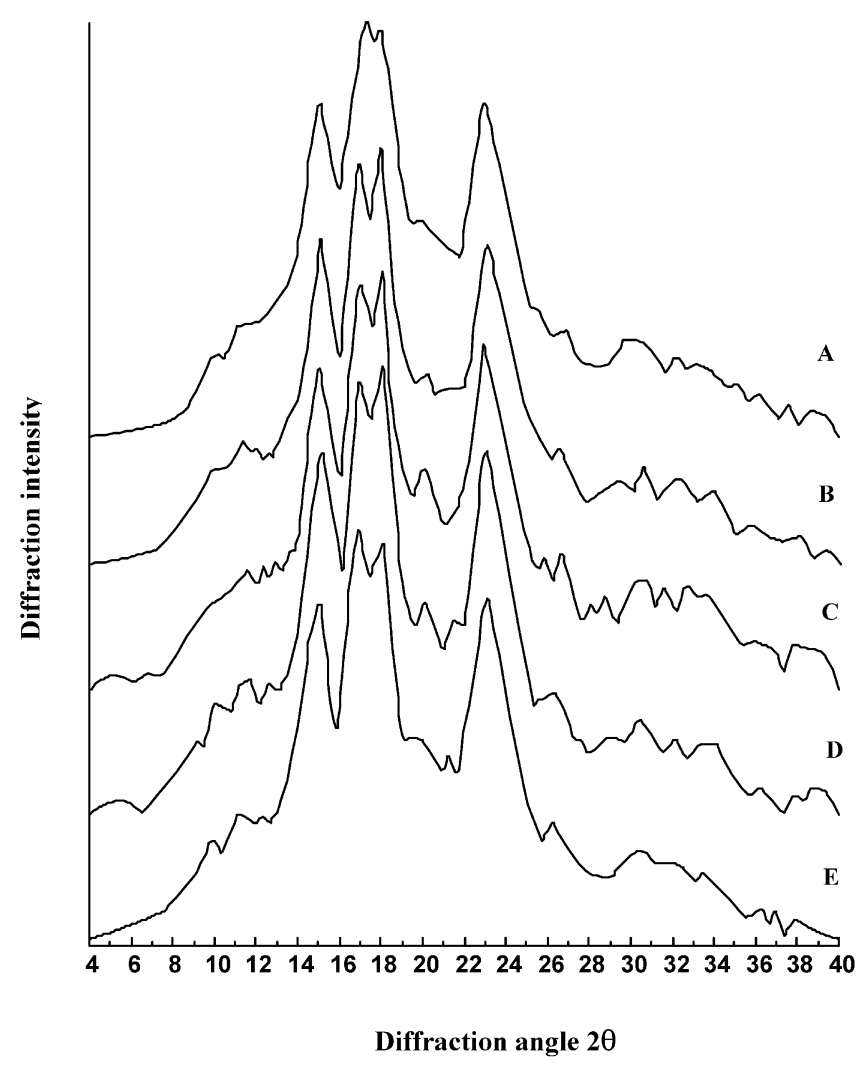

Fig. 1. X-ray diffraction patterns of quinoa starch granules. A, Real; B, Sajama; C, Kanccola; D, Blanca; E, Rosada.

Table 1. Properties of amylose molecules from quinoa starches.

\begin{tabular}{lccccc}
\hline Variety & $\lambda \max ^{\mathrm{a}}$ & $\mathrm{BV}^{\mathrm{b}}$ & $\mathrm{DPn}^{\mathrm{c}}$ & $\mathrm{CL}^{\mathrm{d}}$ & $\mathrm{NC}^{\mathrm{e}}$ \\
\hline Real & 649 & 1.047 & 1054 & 115 & 8 \\
Sajama & 648 & 0.998 & 910 & 99 & 8 \\
Kanccola & 650 & 1.035 & 989 & 98 & 9 \\
Blanca & 649 & 1.101 & 864 & 119 & 6 \\
Rosada & 648 & 1.083 & 822 & 112 & 6 \\
\hline
\end{tabular}

Values represent the mean of three separate measurements.

${ }^{\mathrm{a}}$ Maximum absorption wavelength (nm), ${ }^{\mathrm{b}}$ Blue value at $680 \mathrm{~nm},{ }^{\mathrm{c}}$ Number-average degrees

of polymerization, ${ }^{\mathrm{d}}$ Average chain-length, ${ }^{\mathrm{e}}$ Average number of chains per molecule.

Table 2. Properties of amylopectin molecules from quinoa starches.

\begin{tabular}{lccccc}
\hline Variety & $\lambda \max ^{\mathrm{a}}$ & $\mathrm{BV}^{\mathrm{b}}$ & $\mathrm{DPn}^{\mathrm{c}}$ & $\mathrm{CL}^{\mathrm{d}}$ & $\mathrm{NC}^{\mathrm{e}}$ \\
\hline Real & 590 & 0.196 & 4752 & 20 & 227 \\
Sajama & 590 & 0.190 & 4380 & 20 & 215 \\
Kanccola & 589 & 0.194 & 4199 & 19 & 216 \\
Blanca & 590 & 0.203 & 3403 & 18 & 172 \\
Rosada & 590 & 0.210 & 4300 & 19 & 217 \\
\hline
\end{tabular}

Values represent the mean of three separate measurements.

${ }^{\mathrm{a}}$ Maximum absorption wavelength (nm), ${ }^{\mathrm{b}}$ Blue value at $680 \mathrm{~nm},{ }^{\mathrm{C}}$ Number-average degrees

of polymerization, ${ }^{\mathrm{d}}$ Average chain-length, ${ }^{\mathrm{e}}$ Average number of chains per molecule. 


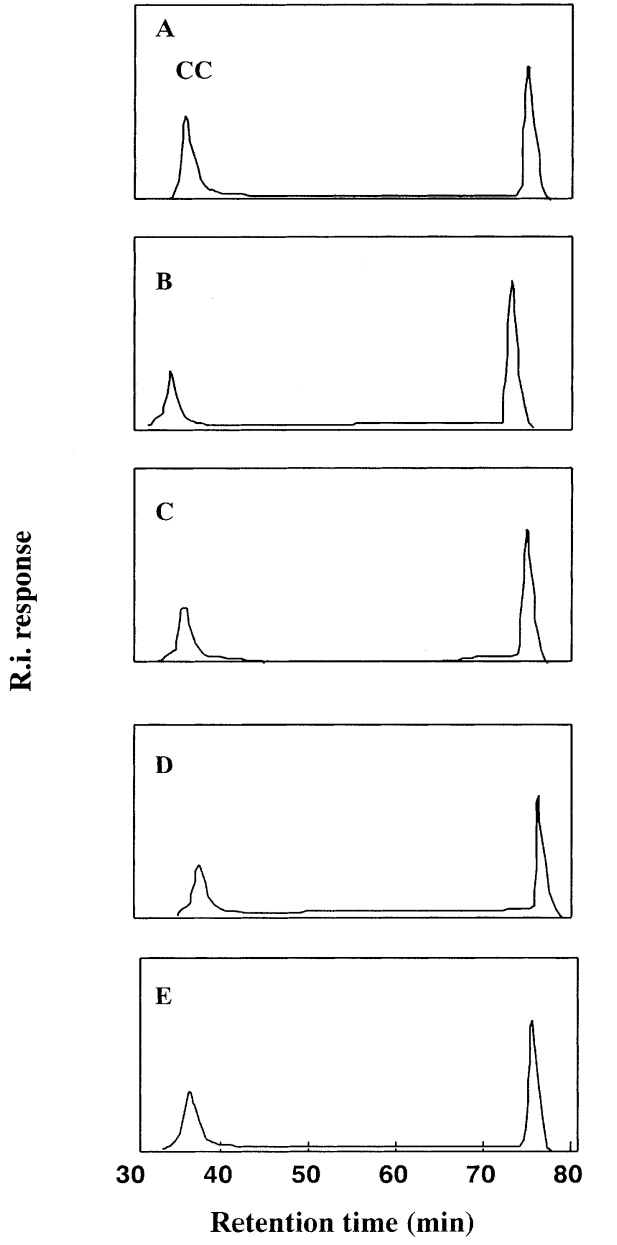

Fig. 2. Elution profiles of isoamylase-debranched amyloses from gel-permeation HPLC.

CC, C-chain; A, Real; B, Sajama; C, Kanccola; D, Blanca; E, Rosada.

the long chain fraction, and F.2 to F.4 were the short chain fractions (Tang et al., 2001a). The average degrees of polymerization of short chains (F.2, F.3, and F.4) were 14.9, 14.9, 15.4, 16.3, and 16.6 for Real, Sajama, Kanccola, Blanca, and Rosada, respectively. The ratios (molar\%) of the short chain fraction (F.2, F.3, and F.4) per long chain fraction (F.1) were 13.9, 14.4, 15.4, 9.7, and 13.3 for the five lines, respectively. It was shown that the average number of short chains per cluster of quinoa amylopectin molecules was 10 to 16 .

\section{Discussion}

Quinoa starch exhibited an A-type of X-ray diffraction pattern (Fig. 1). The degrees of relative crystallinity were 39.0 to $43.0 \%$. Jenkins and Donald (1995) reported that amylose disrupted the structure order within the amylopectin crystallites. Thus, the crystallinity of starch is associated with amylose content. The relative crystallinity of quinoa starch was higher than that of normal barley starch, and similar to that of waxy barley starch (Tang et al., 2002). The amylose content of quinoa starches has been reported to range from 3.0 to $20.0 \%$ (Atwell et al., 1983; Lindeboom et al., 2005). The amylose content of

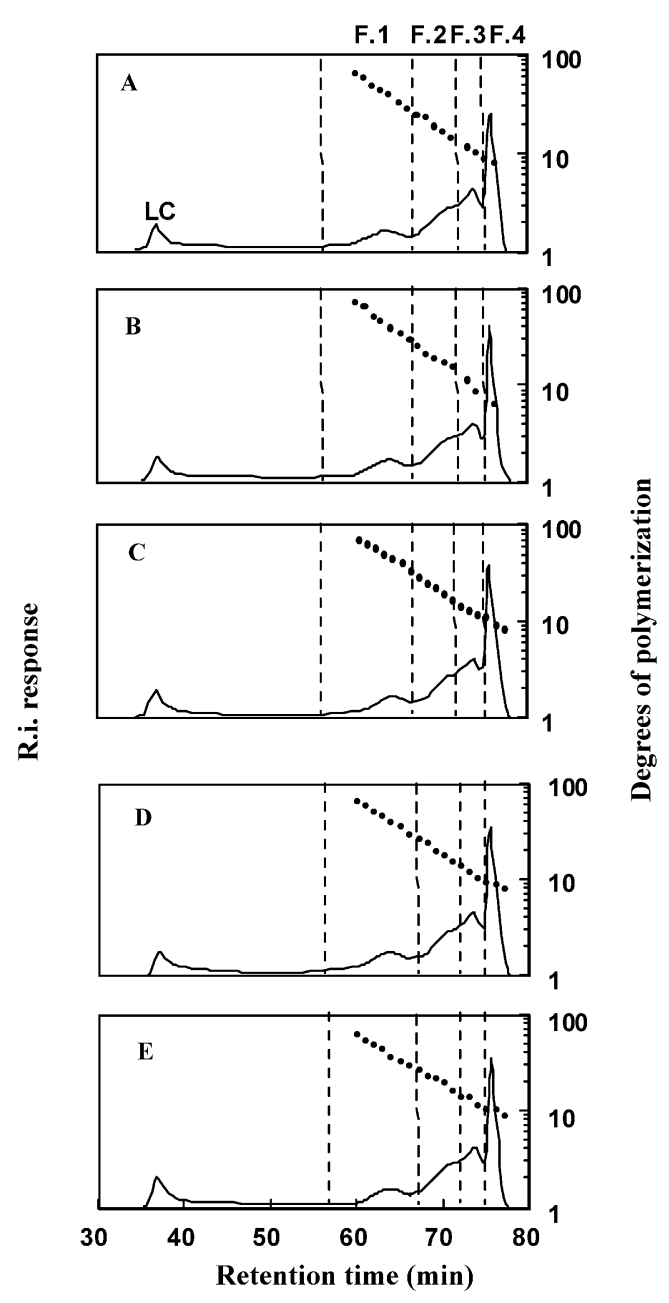

Fig. 3. Elution profiles of isoamylase-debranched amylopectins from gel-permeation HPLC.

— R.i. response; $\bullet$, degrees of polymerization; LC, the longest chain; F.1, fraction 1; F.2, fraction 2; F.3, fraction 3; F.4, fraction 4; A, Real; B, Sajama; C, Kanccola; D, Blanca; E, Rosada.

starches from the five quinoa lines used in this study was 5.2 to $10.9 \%$ (unpublished results), lower than those of normal corn and barley starches, but similar to that of waxy barley starches, and higher than that of waxy corn starches (Tang et al., 2002; Lindeboom et al., 2005). These results showed that no lines with high amylose starch were found among cultivated quinoa, as reported by Lindeboom et al. (2005). The $\lambda \max$ and BV of quinoa amylose (Table 1) were lower than those of barley amylose (Tang et al., 2001a, b). Amylose molecules are composed of linearly linked long glucose polymers, whereas amylopectin molecules have many branched chains of short glucose polymers. Therefore, the $\lambda$ max and BV of amylose are higher than those of amylopectin. Quinoa amylose had a large number of branched chains (Table 1, Fig. 2) compared to barley amylose (Tang et al., 2001a). It was suggested that the low $\lambda$ max and BV of quinoa amylose molecules resulted from the presence of the branched chains. On the other hand, the $\lambda$ max and BV of quinoa amylopectin (Table 2) were higher than those of 
barley amylopectin (Tang et al., 2001a, b). Takeda and Hizukuri (1987) reported that rice amylopectins containing a large proportion of LC showed high $\lambda$ max and BV in a comparison between indica and japonica rice. These results suggest that quinoa amylopectin has a large proportion of LC (Fig. 3) which result in the high values.

The physicochemical properties of starches from various quinoa lines were investigated (Lindeboom et al., 2005). The swelling power of quinoa starches was similar to that of barley and wheat starches (Tang et al., 2002; Tang et al., 2005). However, the freeze thaw stability of quinoa starches was higher than that of barley and wheat starches (Tang et al., 2004; Tang et al., 2005), and the gelatinization onset and peak temperatures of quinoa starches were lower than those of barley starches (Tang et al., 2002). Quinoa starch had different physicochemical and structural properties from barley and wheat starches. Additionally, it was reported that amylopectins with LC were subjected to rapid retrogradation (Takeda and Hizukuri, 1987). These results suggested that quinoa starch was tolerant of freeze thaw, and subjected to rapid retrogradation. Thus, quinoa could be used as a novel food source.

\section{References}

Ahamed, N.T., Singhai, R.S., Kulkarni, P.R. and Pal, M. (1996). Physicochemical and functional properties of Chenopodium quinoa starch. Carbohydr. Poly., 31, 99-103.

Ando, H., Chen, Y.C., Tang, H., Shimizu, M., Watanabe, K. and Mitsunaga, T. (2002). Food components in fractions of quinoa seed. Food Sci.Technol.Res., 8, 80-84.

Atwell, W.A., Patrick, B.M., Johnson, L.A. and Glass R.W. (1983). Characterization of quinoa starch. Cereal Chem., 60, 9-11.

Dubois, M., Gilles, K.A., Hamilton, J.K., Rebers, P.A. and Smith, F. (1956). Colorimetric method for determination of sugars and related substances. Anal. Chem., 28, 350-356.

Hizukuri, S., Takeda, Y., Yasuda, M. and Suzuki, A. (1981). Multibranched nature of amylose and the action of debranching enzymes. Carbohydr. Res., 94, 205-213.

Jenkins, P.J. and Donald, A.M. (1995). The influence of amylase on starch granule structure. Inter. J. Biol. Macromolec., 17, 315-321.

Lindeboom, N., Chang, P.R., Falk, K.C. and Tyler, R.T. (2005).
Characteristics of starch from eight quinoa lines. Cereal Chem., $82,216-222$.

Nara, S., Mori, A. and Komiya, T. (1978). Study on relative crystallinity of moist potato starch. Starch, 30, 111-114.

Ogawa, H., Watanabe, K., Mitsunaga, T. and Meguro, T. (2001). Effect of quinoa on blood pressure and lipid metabolism in diet-induced hyperlipidemic spontaneously hypertensive rats (SHR). J. Jpn. Soc. Nutr. Food Sci., 54, 221-227.

Ranhotra, G.S., Gelroth, J.A., Glaser, B.K., Lorenz, K.J. and Johnson, D.L. (1993). Composition and protein nutritional quality of quinoa. Cereal Chem., 70, 303-305.

Takeda, C., Takeda, Y. and Hizukurim S. (1983). Physicochemical properties of lily starch. Cereal Chem., 60, 212-216.

Takeda, Y., Huzukuri, S. and Juliano, B.O. (1986). Purification and structure of amylose from rice starch. Carbohydr. Res., 148, 299308.

Takeda, Y. and Huzukuri, S. (1987). Structures of rice amylopectins with low and high affinities for iodine. Carbohydr. Res., 168, 79-88.

Takeda, Y., Takeda, C., Mizukami, H. and Hanasuiro, I. (1999). Structures of large, medium and small starch granules of barley grain. Carbohydr. Poly., 38, 109-114.

Tang, H., Ando, H., Watanabe, K., Takeda, Y. and Mitsunaga, T. (2001a). Fine structures of amylose and amylopectin from large medium and small waxy barley starch granules. Cereal Chem., 78, 111-115.

Tang, H., Ando, H., Watanabe, K., Takeda, Y. and Mitsunaga, T. (2001b). Physicochemical properties and structure of large, medium and small granule starches in fractions of normal barley endosperm. Carbohydr. Res., 330, 241-248.

Tang, H., Watanabe, K. and Mitsunaga, T. (2002). Structure and functionality of large, medium and small granule starches in normal and waxy barley endosperms. Carbohydr. Poly., 49, 217224.

Tang, H., Mitsunaga, T. and Kawamura, Y. (2004). Relationship between functionality and structure in barley starches. Carbohydr. Poly., 57, 145-152.

Tang, H., Mitsunaga, T. and Kawamura, Y. (2005). Functionality of starch granules in milling fractions of normal wheat grain. Carbohydr. Poly., 59, 11-17.

Watanabe, K., Ibuki, A., Chen, Y.C., Kawamura, Y. and Mitsunaga, T. (2003). Composition of quinoa protein fractions. J. Jpn. Soc. Food Sci. Technol., 50, 546-549.

Zobel, H.F. (1988). Starch crystal transformations and their industrial importance. Starch, 40, 1-7. 